\title{
Color singlet and adjoint free energy at finite temperature
}

\author{
Alexei Bazavov ${ }^{* a}$, Péter Petreczky ${ }^{b}$, Alexander Velytsky ${ }^{c, d}$ \\ ${ }^{a}$ Department of Physics, University of Arizona, Tucson, AZ 85721, USA \\ ${ }^{b}$ RIKEN-BNL Research Center and Physics Department, Brookhaven National Laboratory, \\ Upton NY 11973, USA \\ ${ }^{c}$ Enrico Fermi Institute, University of Chicago, 5640 S. Ellis Ave., Chicago, IL 60637, USA \\ ${ }^{d}$ HEP Division and Physics Division, Argonne National Laboratory, 9700 Cass Ave., Argonne, IL \\ 60439, USA
}

We study correlation functions of static quark-antiquark pairs in $S U(2)$ gauge theory at finite temperature. By measuring Polyakov loop correlators and temporal Wilson loops with APE smearing of spatial links we are able to give consistent definitions of the singlet and adjoint free energies at short distances, where the notion of these free energies is meaningful. APE smearing procedure allows to achieve a high degree of overlap in the singlet channel and to reconstruct the adjoint part from the color averaged and singlet free energy.

The XXVI International Symposium on Lattice Field Theory

July 14 - 19, 2008

Williamsburg, Virginia, USA

${ }^{*}$ Speaker. 


\section{Introduction}

Strongly interacting matter undergoes a deconfining transition at some temperature which is triggered by large increase in the number of degrees of freedom as well as melting of hadronic states. Another important feature of the deconfined phase is the screening of color charges. It has been argued that color screening will lead to quarkonium dissociation above the deconfinement temperature which can be used as a signature of quark gluon plasma formation in heavy ion collisions [1]. Melting of quarkonium states has been studied in potential models with screened potentials (see e.g. [2] and references therein). Alternatively, one may try to reconstruct quarkonium spectral functions from Euclidean correlators (see e.g. [3, 丹] and references therein). It turns out, however, that quarkonium melting does not affect the Euclidean meson correlators and these correlators are almost temperature independent. This is contrary to what happens in the light quark sector, where both meson correlators and spectral functions show significant temperature dependence in the deconfined phase [5]. Therefore potential models are useful tools to study in-medium quarkonium properties. But to establish their applicability a better understanding of color screening is needed.

On the lattice color screening is usually studied in terms of the Polyakov loop correlator related to the free energy of static quark anti-quark pair [6]. This correlator shows significant temperature dependence across the transition and in the deconfined phase the free energy of static quark antiquark pair shows large temperature dependence even for very small separations between the static quark and anti-quark, much smaller than the inverse temperature. In perturbative picture this can be understood due to the fact that in the deconfined phase not only singlet quark anti-quark $(Q \bar{Q})$ states contribute to the free energy but also colored states with $Q \bar{Q}$ in the adjoint representation. This observation is also supported by lattice calculations of the correlation function of two temporal Wilson lines in Coulomb gauge, which in perturbation theory corresponds to the so-called singlet free energy [9]. The singlet free energy is temperature independent at short distances and coincides with the zero temperature potential as expected. At large distances, however, it also shows significant temperature dependence.

The problem of defining color singlet and adjoint $Q \bar{Q}$ states on the lattice has been considered in Ref. [7]. It has been found that the conventional definition of singlet and adjoint states have problems. Here we report on our study [8] of static meson correlators in 4 dimensional $S U(2)$ gauge theory at finite temperature and show how the problem observed in Ref. [7] can be resolved in the limit of small distances and/or high temperatures.

\section{Static meson correlators}

On the lattice correlators of the static meson operators in color singlet and adjoint states at $t=1 / T$ have the form [元]:

$$
\begin{gathered}
G_{1}(r, T)=\frac{1}{N}\left\langle\operatorname{Tr} L^{\dagger}(x) U(x, y ; 0) L(y) U^{\dagger}(x, y, 1 / T)\right\rangle, \quad r=|x-y|, \\
G_{a}(r, T)=\frac{1}{N^{2}-1}\left\langle\operatorname{Tr} L^{\dagger}(x) \operatorname{Tr} L(y)\right\rangle-\frac{1}{N\left(N^{2}-1\right)}\left\langle\operatorname{Tr} L^{\dagger}(x) U(x, y ; 0) L(y) U^{\dagger}(x, y, 1 / T)\right\rangle,
\end{gathered}
$$


where $N$ is the number of colors ( $N=2$ in our numerical calculations). Here $L(x)$ is the temporal Wilson line, which on the lattice is simply $L=\prod_{\tau=0}^{N_{\tau}-1} U_{0}(x, \tau)$ with $U_{0}(x, \tau)$ being the temporal links. The correlators depend on the choice of the spatial transporters $U(x, y ; t)$. In the special gauge, where $U(x, y ; t)=1$ the above correlators give standard definition of the singlet and adjoint free energies

$$
e^{-F_{1}(r, T) / T}=\frac{1}{N}\left\langle\operatorname{Tr} L^{\dagger}(x) L(y)\right\rangle, \quad e^{-F_{a}(r, T) / T}=\frac{\left\langle\operatorname{Tr} L^{\dagger}(x) \operatorname{Tr} L(y)\right\rangle}{N^{2}-1}-\frac{\left\langle\operatorname{Tr} L^{\dagger}(x) L(y)\right\rangle}{N\left(N^{2}-1\right)} .
$$

The singlet and triplet free energies can be calculated at high temperature in leading order HTL approximation [9]. At this order $F_{1}$ and $F_{a}$ are gauge independent or in other words do not depend on the choice of the parallel transporters $U(x, y ; t)$. At small distances the singlet free energy is temperature independent and coincides with the zero temperature potential, while the adjoint free energy depends on the temperature [9].

The physical free energy of a static quark anti-quark pair is given by the thermal average of the singlet and adjoint free energies and is explicitly gauge independent

$$
e^{-F(r, T) / T}=\frac{1}{N^{2}} e^{-F_{1}(r, T) / T}+\frac{N^{2}-1}{N^{2}} e^{-F_{a}(r, T) / T}=\frac{1}{N^{2}}\langle\operatorname{Tr} L(x) \operatorname{Tr} L(y)\rangle \equiv \frac{1}{N^{2}} G(r, T) .
$$

Using the transfer matrix one can show that in the confined phase [7]

$$
G_{1}(r, T)=\sum_{n=1}^{\infty} c_{n}(r) e^{-E_{n}(r, T) / T}, \quad G(r, T)=\sum_{n=1}^{\infty} e^{-E_{n}(r, T) / T},
$$

where $E_{n}$ are the energy levels of static quark and anti-quark pair. The coefficients $c_{n}(r)$ depend on the choice of the gauge transporters. The color averaged correlator $G(r, T)$ does not contain $c_{n}$. The lowest energy level is the usual static quark anti-quark potential, while the higher energy levels correspond to hybrid potentials [10]. If $c_{1}=1$ the dominant contribution to $G_{a}$ would be the first excited state $E_{2}$, i.e. the lowest hybrid potential which at short distances is related to the adjoint potential. In this sense $G_{a}$ is related to static mesons with $Q \bar{Q}$ in adjoint state. Numerical calculations show, however, that $c_{1}(r) \neq 1$ and depends on the separation $r$. Thus $G_{a}$ also receives contribution from $E_{1}$ [汤. The lattice data seem to suggest that $c_{1}$ approaches unity at short distances in accord with expectations based on perturbation theory, where $c_{1}=1$ up to $\mathscr{O}\left(g^{6}\right)$ corrections [11]. Therefore at short distances, $r \ll 1 / T$ the color singlet and color averaged free energy are related $F(r, T)=F_{1}(r, T)+T \ln \left(N^{2}-1\right)$. This relation is indeed confirmed by lattice calculations [12].

\section{Numerical results}

We have calculated correlation functions of static mesons $G_{1}(r, T)$ and $G(r, T)$ both in the confined and deconfined phase of $S U(2)$ gauge theory. The details of the calculations are presented in Ref. [8]. We have studied the color singlet and averaged correlators given by Eqs. (2.1) and (2.4). The spatial links entering the transporter $U(x, y ; 0)$ were smeared using APE smearing, which has been applied iteratively. The weight of the staple in the APE smeared link was 0.12. For $\beta=2.5$ we use spatial links with 10 steps of APE smearing and unsmeared spatial links. For $\beta=2.7$ we used unsmeared spatial links as well as spatial links with 10 steps and 20 steps of APE smearing. 


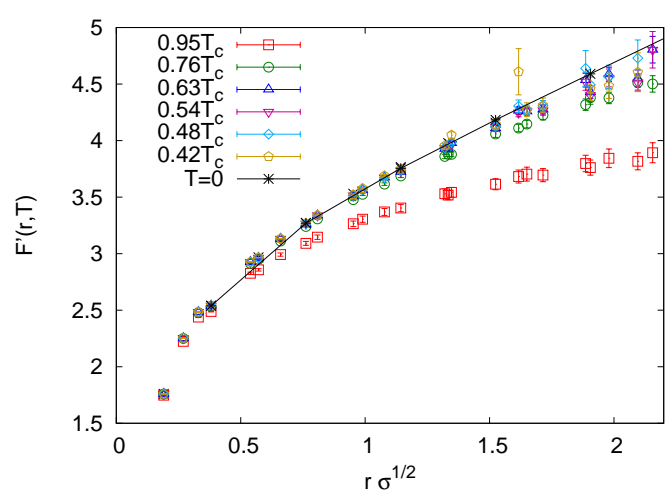

Figure 1: The color averaged free energy below deconfinement temperature at $\beta=2.5$ calculated on $32^{3} \times N_{\tau}$ lattices. Also shown is the $T=0$ potential.

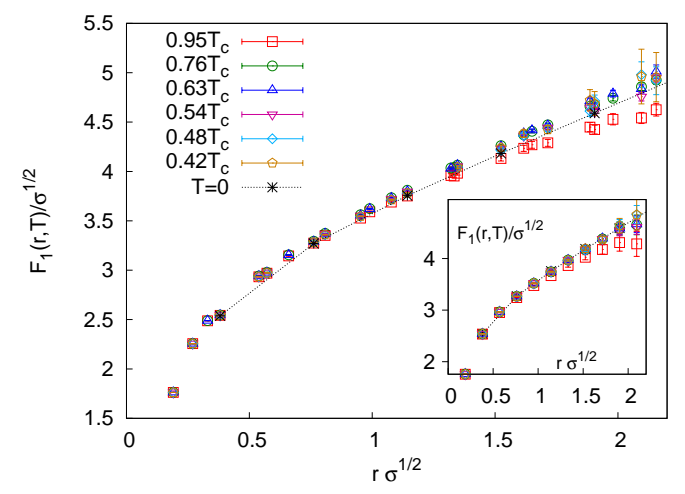

Figure 2: The color singlet free energy below deconfinement temperature at $\beta=2.5$ calculated on $32^{3} \times N_{\tau}$ lattices. It is also shown with the contribution $T \ln c_{1}$ subtracted (inset).

\subsection{Color averaged correlator in the confined phase}

The color averaged correlator has been calculated in the confined phase in the temperature interval $0.32 T_{c}-0.95 T_{c}$ for $\beta=2.5$ and $0.49 T_{c}-0.98 T_{c}$ for $\beta=2.7$. The numerical results for the color averaged free energy for $\beta=2.5$ are shown in Figure 11. To eliminate the trivial temperature dependence due to the color trace normalization in Figure 1 we show the subtracted free energy $F^{\prime}(r, T)=F(r, T)-T \ln 4$ together with zero temperature potential. The color averaged free energy does not show any temperature dependence up to temperatures of about $0.76 T_{c}$. Since the temperature dependence for $T<0.76 T_{c}$ is relatively small we attempted to fit the color averaged correlator with the 1-exponential form $G(r, T)=c_{1}^{a}(r) \exp \left(-E_{1}(r) / T\right)$. The ground state energy $E_{1}(r)$ extracted from this fit agrees well with the zero temperature potential calculated in Ref. [10], while the coefficients $c_{1}^{a}(r)$ are close to one as expected.

\subsection{Color singlet correlators in the confined phase}

The color singlet correlators have been calculated using different levels of APE smearing in the spatial gauge connection. We have found that when no smearing is used the color singlet free energy, $-T \ln G_{1}(r, T)$ shows a small but visible temperature dependence. In particular $F_{1}(r, T)$ is larger than the $T=0$ potential for intermediate distances $0.5<r \sqrt{\sigma}<2$. The temperature dependence of the singlet free energy is significantly reduced when APE smearing is applied. In Figure 2 we show the color singlet free energy for $\beta=2.5$ and 10 APE smearings. As one can see from the figure the color singlet free energy shows much smaller temperature dependence as we get closer to the deconfinement temperature.

To understand the temperature dependence of the color singlet correlator we use 1-exponential fit $G_{1}(r, T)=c_{1}(r) \exp \left(-E_{1}(r) / T\right)$. In all cases considered the values of $E_{1}(r)$ extracted from fits are in good agreement with the calculation of the zero temperature potential in Ref. [10]. The value of the prefactor $c_{1}(r)$ is shown in Figure 3. When no APE smearing is used the value of $c_{1}(r)$ strongly depends on the separation $r$. At small distances it shows a tendency of approaching unity as one would expect in perturbation theory and decreases with increasing distance $r$. At large distance 

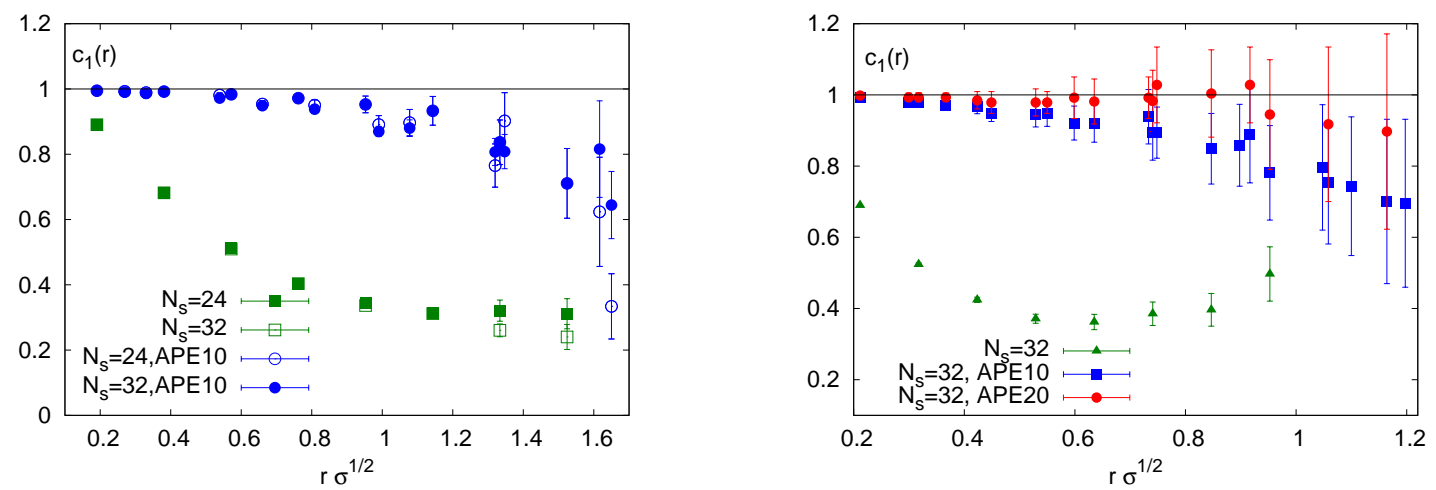

Figure 3: The pre-exponential factor of the color singlet correlators as function of distance $r$ for $\beta=2.5$ (left) and $\beta=2.7$ (right). Shown are results for unsmeared spatial links and 10 and 20 steps of APE smearing.

its value is around $0.3-0.5$. Similar results for $c_{1}$ have been obtained in study of $S U(2)$ gauge theory in 3 dimensions [汤. When APE smearing is applied the $r$-dependence of the amplitude $c_{1}$ is largely reduced and its value is close to unity both for $\beta=2.5$ and $\beta=2.7$. For $\beta=2.7$ we also see that increasing the number of smearing steps from 10 to 20 reduces the deviation of $c_{1}$ from unity. In any case at sufficiently short distances $c_{1}$ is very close to one as expected in perturbation theory. Thus, almost the entire temperature dependence of the singlet free energy at distances $0.5<r \sqrt{\sigma}<2$ is due to the deviation of $c_{1}$ from unity and can be largely reduced by applying APE smearing to the links in the spatial gauge connections. To further demonstrate this point in the inset of Figure 2 we show the results for $F_{1}(r, T)+T \ln c_{1}(r)$.

\subsection{Color singlet free energy in the deconfined phase}

It turns out that the singlet free energy, $F_{1}(r, T)=-T \ln G_{1}(r, T)$, calculated from cyclic Wilson loops shares the same qualitative features as the singlet free energy calculated in Coulomb gauge [13]. At short distances it is temperature independent and coincides with the zero temperature potential. At large distances it approaches a constant $F_{\infty}(T)$, which is the free energy of two isolated static quarks at infinite separation.

At leading order $F_{1}(r, T)-F_{\infty}(T)$ is of Yukawa form, therefore in Figure $\square$ we show our numerical results in terms of the screening function $S(r, T)=r \cdot\left(F_{1}(r, T)-F_{\infty}(T)\right)$ at different temperatures. At short distances $(r T<0.5)$ the singlet free energy does not depend on the smearing level. Furthermore, it is very close to the free energy calculated in Coulomb gauge. We expect that at large distances the screening function $S(r, T)$ will show an exponential decay determined by a temperature dependent screening mass $m_{1}(T)$, which is equal to the leading order Debye mass up to the non-perturbative $g^{2}$ corrections: $m_{1}=m_{D}+\mathscr{O}\left(g^{2}\right)$. From Fig. 7 we can see that indeed $S(r, T)$ behaves exponentially with screening mass proportional to the temperature. Fitting the large distance behavior of the screening function by an exponential form $\exp \left(-m_{1}(T) r\right)$ we determine the screening mass $m_{1}(T)$. In Fig. $\emptyset$ we also show the color singlet screening masses extracted from the fits and compare them to the results obtained in Coulomb gauge in Ref. [13] as well as to the leading order Debye mass calculated using 2-loop gauge coupling $g(\mu=2 \pi T)$ in $\overline{M S}$-scheme. As we see from the figure the screening masses are smaller than those calculated in Coulomb gauge 


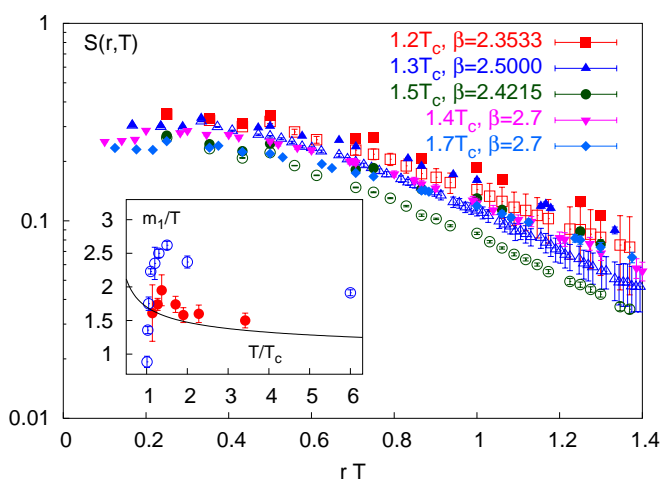

Figure 4: The screening function at different temperatures as function of $r T$ and different values of $\beta$. In the inset the screening masses $m_{1}$ extracted from singlet free energies are shown. The line shows the leading order results for the Debye mass.

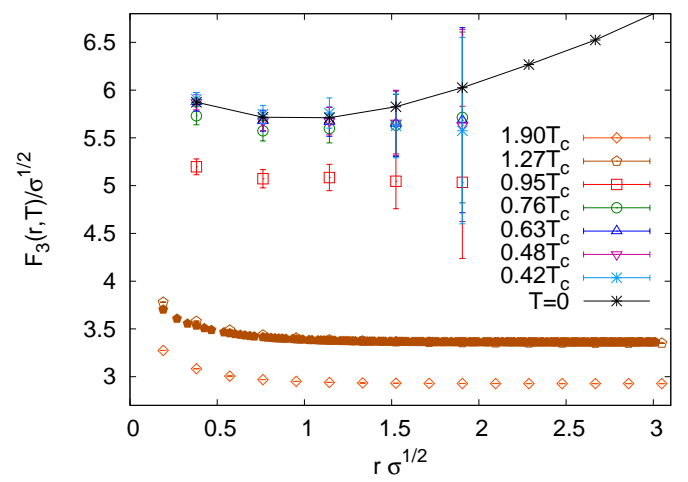

Figure 5: The triplet free energy at different temperatures calculated at $\beta=2.5$. The filled symbols correspond to calculations in Coulomb gauge. Also shown is the first hybrid potential calculated in Ref. [10].

and agree well with the leading order perturbative prediction.

\subsection{Color triplet free energy}

We have calculated the color triplet correlator defined by Eq. (2.2) for different temperatures below and above the transition temperature. Below the deconfinement temperature we observe a moderate $T$-dependence of the triplet correlator. We also find that the corresponding free energy $-T \ln G_{3}(r, T)$ is smaller than the first hybrid potential calculated in Ref. [10], but larger than the triplet free energy in Coulomb gauge [13].

Let us assume that only two states contribute to the Eqs. (2.5). Then from Eq. (2.2) it follows that

$$
F_{3}(r, T)=E_{2}(r)-T \ln \left(1-c_{2}(r)+\frac{1}{3}\left(1-c_{1}(r)\right) e^{\Delta E(r) / T}\right),
$$

with $\Delta E(r)=E_{2}(r)-E_{1}(r)$ and $c_{2} \ll 1$. We have subtracted the correction $T \ln \left(1+\frac{1}{3}\left(1-c_{1}\right) e^{\Delta E / T}\right)$ from the triplet free energy assuming that $E_{1}(r)$ is given by the ground state potential and $E_{2}(r)$ is given by the first hybrid potential as calculated in Ref. [10]. The numerical results are summarized in Fig. 5 which shows that after this correction is accounted for in the confined phase the triplet free energy at low temperatures agrees reasonably well with the first hybrid potential. As temperature increases more excited states contribute. In particular, at $0.76 T_{c}$ the value of the triplet free energy can be accounted for by including the next hybrid state [10].

\section{Conclusions}

We have studied singlet and triplet static quark anti-quark correlators in finite temperature $S U(2)$ theory expressed in terms of Polyakov loop correlators and cyclic Wilson loops. In leading order and probably next-to-leading order of perturbation theory the static correlators defined by Eq. (2.1) and (2.2) project onto singlet and triplet states respectively, however, this separation does not hold in general case. Due to interactions with ultrasoft fields there will be a mixing of 
singlet and triplet states which is proportional to $\alpha_{s}^{3}(1 / r)$ and $(r \mu)^{4}$, with $\mu$ being the ultrasoft scale [11]. In our case the ultrasoft scale can be the binding energy, $\alpha_{s} / r, \Lambda_{Q C D}$ or $g^{2} T$. Therefore it is expected that mixing is quite small at sufficiently small distances. We determined the mixing between singlet and triplet states in terms of the overlap factor $c_{1}(r)$. If the overlap factor is unity there is no mixing. Our lattice calculations show that $c_{1}$ indeed approaches one at small distances. Therefore the contribution of singlet state to $G_{3}(r, T)$ appears to be small at temperatures close to deconfinement temperature. This contribution is also controlled by the non-perturbative gap between the singlet and triplet states, i.e. the gap between the static potential and the first hybrid potential.

Our analysis shows that at short distances $r T<1$ the singlet correlator is almost temperature independent, while the triplet correlator is largely affected by the deconfinement. The temperature dependence of the triplet correlators indicate the melting of the non-perturbative gap between the singlet and the triplet states above deconfinement, which turns out to be consistent with perturbative expectations. This finding is important for application of thermal pNRQCD discussed in Ref. [14] to realistic quarkonia and temperatures not very far from the deconfinement temperature.

\section{Acknowledgments}

This work was supported by U.S. Department of Energy under Contract No. DE-AC0298CH10886. The work of A.B. was supported by grants DOE DE-FC02-06ER-41439 and NSF 0555397. A.V. work was supported by the Joint Theory Institute funded together by Argonne National Laboratory and the University of Chicago, and in part by the U.S. Department of Energy, Division of High Energy Physics and Office of Nuclear Physics, under Contract DE-AC02$06 \mathrm{CH} 11357$.

\section{References}

[1] T. Matsui and H. Satz, Phys. Lett. B 178, 416 (1986).

[2] Á. Mócsy and P. Petreczky, Phys. Rev. D 77, 014501 (2008); Phys. Rev. Lett. 99, 211602 (2007).

[3] A. Jakovac, P. Petreczky, K. Petrov and A. Velytsky, Phys. Rev. D 75, 014506 (2007).

[4] S. Datta, F. Karsch, P. Petreczky and I. Wetzorke, Phys. Rev. D 69, 094507 (2004).

[5] F. Karsch, E. Laermann, P. Petreczky, S. Stickan and I. Wetzorke, Phys. Lett. B 530, 147 (2002).

[6] L. D. McLerran and B. Svetitsky, Phys. Rev. D 24, 450 (1981).

[7] O. Jahn and O. Philipsen, Phys. Rev. D 70, 074504 (2004).

[8] A. Bazavov, P. Petreczky and A. Velytsky, [arXiv:0809.2062v1 [hep-lat]].

[9] P. Petreczky, Eur. Phys. J. C 43, 51 (2005).

[10] C. Michael, S.J. Perantonis, J. Phys. G 18, 1725 (1992).

[11] N. Brambilla, A. Pineda, J. Soto and A. Vairo, Nucl. Phys. B 566, 275 (2000).

[12] O. Kaczmarek, F. Karsch, P. Petreczky and F. Zantow, Phys. Lett. B 543, 41 (2002).

[13] S. Digal, S. Fortunato and P. Petreczky, Phys. Rev. D 68, 034008 (2003).

[14] N. Brambilla, J. Ghiglieri, A. Vairo and P. Petreczky, Phys. Rev. D 78, 014017 (2008). 